\title{
Monotone Systems Under Positive Feedback: Multistability and a Reduction Theorem
}

\author{
German Enciso $^{\text {a,1 }}$ Eduardo D. Sontag ${ }^{\text {a, }}{ }^{2}$

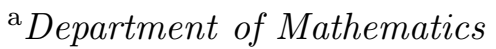 \\ Rutgers University, New Brunswick, NJ 08903
}

\begin{abstract}
For feedback loops involving single input, single output monotone systems with well-defined I/O characteristics, a recent paper by Angeli and Sontag provided an approach to determining the location and stability of steady states. A result on global convergence for multistable systems followed as a consequence of the technique. The present paper extends the approach to multiple inputs and outputs. A key idea is the introduction of a reduced system which preserves local stability properties.
\end{abstract}

Key words: monotone systems, unity feedback, global stability

\section{Introduction}

The study of stability properties for systems having multiple steady states is of great importance, as such systems possess a "memory" of past states, and, as components of larger systems, can act as switches, or underlie relaxation oscillators. A result on global convergence for multistable systems was presented in the article [2]. That work presented a reduction principle for unity feedback loops involving single input, single output models which admit a well-defined $\mathrm{I} / \mathrm{O}$ characteristic and satisfy a monotonicity condition. The reduction was to a discrete-time one-dimensional iteration.

1 E-mail: enciso@eden.rutgers.edu.

Supported in part by AFOSR Grant F49620-01-1-0063, NIH Grant P20 GM64375, and Dimacs.

2 E-mail: sontag@control.rutgers.edu.

Supported in part by AFOSR Grant F49620-01-1-0063 and NIH Grant R01 GM46383. 
This note is a follow-up, where we extend the result to the multivariable case. The key idea is to introduce a continuous-time reduced system which preserves local stability properties. This reduced system is often easier to analyze than the original one, since its dimension equals the number of inputs (or outputs), which is typically very small. Model reduction is a powerful analysis tool in differential equations and control theory. The two most used approaches are those involving energy considerations, where only the "largest" components, as measured in an appropriate norm, are kept (see e.g. [5]), and classical timescale separation (singular perturbation techniques). The approach proposed in [2], and extended here, is completely different to these two.

In this paper, we deal with systems with inputs and outputs, as usual in control theory $([17])$ :

$$
\dot{x}=f(x, u), y=h(x),
$$

with $u(t) \in U$ and $y(t) \in Y$, but we assume that the input-value and outputvalue spaces coincide $\left(U=Y\right.$ is a subset of some Euclidean space $\mathbb{R}^{m}$; states evolve in some subset $X \subseteq \mathbb{R}^{n}$ ). We assume that $U=Y$ because our goal is to analyze the stability properties of the closed loop system

$$
\dot{x}=f(x, h(x))
$$

which arises under unity feedback. More general feedback interconnections can be reduced to a unity feedback configuration for purposes of applying these techniques (see for instance the discussion in [3]). We illustrate with an example how to rewrite a given system in a form suitable for application of our theory.

The main assumptions are that the open-loop system (1) is monotone with respect to cones $\mathcal{K}_{m}, \mathcal{K}_{n}$, and $\mathcal{K}_{m}$ in the input-value, state, and output-value spaces, and that it admits a nondegenerate I/S characteristic $k^{X}: U \rightarrow X$. We denote the corresponding (nondegenerate) I/O characteristic as $k=h \circ k^{X}$ : $U \rightarrow U$. In our main result we will establish a connection between (1) and the reduced system:

$$
\dot{u}=k(u)-u .
$$

Theorem 1 Let (1) be a monotone system that admits a nondegenerate I/S characteristic $k^{X}$ and an I/O characteristic $k$ with nondegenerate fixed points, and assume that the closed loop system (2) is strongly monotone. Then the function $\bar{u} \rightarrow k^{X}(\bar{u})$ forms a bijective correspondence between the locally asymptotically stable points of the monotone system $\dot{u}=k(u)-u$ and those of (2). Furthermore, almost all bounded solutions of (2) converge to one of these asymptotically stable points. 
This reduction allows to study the global stability properties of the full system (1) in terms of the reduced system, which has, in general, much lower dimensionality than (1). In [2], a result was proved that is equivalent to this one for the special case of scalar inputs and outputs $(m=1)$. This scalar result was formulated in terms of a discrete-time condition involving derivatives of $k$. One of the main contributions of the present paper is the re-interpretation of that condition in terms of the reduced-order continuous-time system (3). This re-interpretation is crucial to the generalization that we gave in Theorem 1 . Theorems 1 and 2 are the exact counterparts of Theorem 3 and Lemma 6.6 of [2], respectively, even though the statements are written somewhat differently. The main theorem is of use in a number of applications, especially in biological signaling networks with multiple steady states and/or presenting hysteresis effects; see [1-4,18].

One of the most interesting implications of this methodology lies in the fact that the mapping $k$ can be often obtained from experimental data ("dose response" curves in pharmacology, for example), even when knowledge of the system (1) is poor because of uncertainty in the form of equations, or unknown or unmeasurable parameters. Provided that general qualitative knowledge about the system is available (insuring the appropriate assumptions for the system to apply), one can then mathematically conclude stability from the input/output data provided by $k$. This is discussed in detail in $[3,18]$.

The organization of this paper is as follows. After stating some basic definitions, we first establish a number of preliminary results about positive matrices, followed by the local version of the result (linear systems). Theorem 1 will then follow by combining this local result with a global convergence theorem due to Hirsch. After this, we discuss an example, and we study sufficient conditions for closed-loops to be strongly monotone.

\section{Definitions}

Let $\mathcal{K} \subseteq \mathbb{R}^{n}$ be a cone, by which we mean a set that is nonempty, convex, closed under multiplication by positive scalars, and pointed (i.e. $\mathcal{K} \cap(-\mathcal{K})=\{0\}$ ). We will also assume that $\mathcal{K}$ is closed and has nonempty interior (it is "proper"). The cone $\mathcal{K}$ induces the partial order given by: $x \leq y$ iff $y-x \in \mathcal{K}$, and the stronger order $x \ll y$ iff $y-x \in \operatorname{int} \mathcal{K}$. We also say that $x<y$ if $x \leq y$ and $x \neq y$.

Assume given a system (1), where the state space $X \subseteq \mathbb{R}^{n}$ is the closure of an open set, the input- and output-value set $U$ is also the closure of an open set, and $f$ and $h$ are continuously differentiable. We also assume given two proper cones $\mathcal{K}_{n} \subset \mathbb{R}^{n}$ and $\mathcal{K}_{m} \subseteq \mathbb{R}^{m}$. By an input we mean a measurable essentially bounded map $u: \mathbb{R}^{+} \rightarrow U$ and write " $u \leq v$ " for two inputs provided that 
$u(t) \leq v(t)$ for almost all $t$. (We abuse notation and use letters such as $u$ to denote both an input value -element of $U$ - or an input, depending on the context.)

The system (1) is monotone with respect to $\mathcal{K}_{n}, \mathcal{K}_{m}$ if $h$ is a monotone function, that is $x \leq y$ implies $h(x) \leq h(y)$, and the flow preserves the order, i.e., the following property is satisfied:

For any two inputs $u, v$ such that $u \leq v$, and any two initial conditions $x_{1}, x_{2} \in X$ such that $x_{1} \leq x_{2}$, it holds that $x\left(t, x_{1}, u\right) \leq x\left(t, x_{2}, v\right)$ for all $t \geq 0$.

Here, $x\left(t, x_{0}, u\right)$ is the solution of the system (1) with initial condition $x_{0}$, evaluated at time $t$, and the relations $\leq$ are defined as in the previous section for each cone, and interpreted as $\leq_{U}$ or $\leq_{X}$ in the obvious manner. Systems with no inputs can be seen as a particular case (using an input value space consisting of just one point); such a system is monotone if $x_{1} \leq x_{2}$ implies $x\left(t, x_{1}\right) \leq x\left(t, x_{2}\right)$ for all $t$. We will always understand "for all $t$ " to mean for all times $t$ belonging to the common domain of definition of the solutions involved.

A system of the form (1) is said to be strongly monotone if $u \leq v, x_{1}<x_{2}$ implies $x\left(t, x_{1}, u\right) \ll x\left(t, x_{2}, v\right) \forall t$. We also assume in this case that $x \ll y$ implies $h(x) \ll h(y)$ (we say that $h$ itself is strongly monotone).

It is useful to be able to test monotonicity directly in terms of vector fields. In [1], two characterizations are provided, one in terms of nonsmooth analysis and valid for abstract monotone dynamics (even with respect to arbitrary partial orders), and a second one, quoted next, based upon a generalization to systems with inputs of the concept of quasi-monotonicity, the latter of which was introduced by Schneider and Vidyasagar, and used later by Volkmann (see $[14,20])$. The system (1) is monotone if and only if

$$
\begin{array}{r}
x \in X, u \in U, h \in \mathcal{K}_{n}, v \in \mathcal{K}_{m}, \phi \in \mathcal{K}_{n}^{*}, \text { and } \sigma(h)=0 \\
\Rightarrow \sigma(f(x+h, u+v)-f(x, u)) \geq 0
\end{array}
$$

where $\mathcal{K}_{n}^{*}=\left\{\sigma \in\left(R^{n}\right)^{*} \mid \sigma(x) \geq 0 \forall x \geq 0\right\}$.

When the state space and input value space are convex, we can rewrite this condition as follows:

$$
\begin{array}{r}
x \in X, u \in U, h \in \mathcal{K}_{n}, v \in \mathcal{K}_{m}, \sigma \in \mathcal{K}_{n}^{*}, \text { and } \sigma(h)=0 \\
\Rightarrow \sigma\left(f_{x}(x, u) h+f_{u}(x, u) v\right) \geq 0
\end{array}
$$


where $f_{x}$ and $f_{u}$ denote the Jacobians of $f$ with respect to the $x$ and $u$ variables respectively (see [13] for an analogous observation for systems with no inputs). As an illustration, we show that the autonomous system (3) is monotone, where $k: U \rightarrow U$ is any monotone function. By condition (4), it is enough to note the following. If $u \leq v$, and $\sigma \in \mathcal{K}^{*}$ such that $\sigma(u)=\sigma(v)$, then

$$
\sigma(k(v)-v)-\sigma(k(u)-u)=\sigma(k(v)-k(u)) \geq 0
$$

\subsection{Characteristics}

We say that (1) has a well-defined input to state characteristic $k^{X}: U \rightarrow X$ if for every constant input $u(t) \equiv u \in U, x\left(t, x_{0}, u\right)$ converges to $k^{X}(u)$ for every initial condition $x_{0} \in X$. In that case we refer to $k=h \circ k^{X}$ as the system's input to output characteristic. We will also assume throughout in this text that the characteristic $k^{X}$ is nondegenerate, that is, $\operatorname{det} f_{x}\left(k^{X}(u), u\right) \neq 0$ for every $u \in U$.

We say that $k$ has nondegenerate fixed points if $\operatorname{det}\left(k^{\prime}(\bar{u})-I\right) \neq 0$ (i.e., $k^{\prime}(u)$ has no eigenvalues equal to one) for each $\bar{u}$ fixed point of $k$ (not to confuse with the previous definition).

Suppose given a system (1) which is both monotone and admits an I/O characteristic $k$. Then $k$ is a monotone function. This is proved as follows. Pick any two elements $u \leq v$ in $U$, and consider the corresponding constant inputs $u(t) \equiv u$ and $v(t) \equiv v$ as well as an arbitrary initial state $x_{0}$. By monotonicity, $x\left(t, x_{0}, u\right) \leq x\left(t, x_{0}, v\right)$. Letting $t$ tend to infinity, we conclude that $k(u) \leq k(v)$.

We finally turn to the question of showing that system (3) has well defined and unique solutions. Note that the function $k^{X}$ is defined as a level curve of $f(x, u)$, where the latter function can be extended in the first variable to an open domain covering $X \times U$, from the definition of differentiability on a closed domain. By the implicit function theorem, and using the nondegeneracy hypothesis, we have that $k^{X}$ itself is a continuously differentiable function on the state space, boundary points included. Composing with the output function $h$, after applying similar remarks, we see that $k(u)$ is $C^{1}$ on $U$ and its boundary points. Thus system (3) has unique, maximally defined solutions.

\section{Preliminaries on Positive Matrices}

We begin with a definition. Given a cone $\mathcal{K} \subseteq \mathbb{R}^{n}$, an $n \times n$ matrix $A$ is called inverse-positive with respect to $\mathcal{K}$ if for every $x \in \mathbb{R}^{n}, A x \geq 0$ implies 
$x \geq 0$. We note first that an inverse-positive matrix $A$ must be invertible: if $A x=0$, then also $A(-x)=0$, which implies $x \in K,-x \in K$, and $x=0$. Also, it is easy to see that $A^{-1} K \subseteq K$, and that these two conditions imply inverse-positivity.

We quote the following results without proof from Plemmons et al, [6], p. 113 and p. 10 respectively. (That reference also calls inverse positive matrices $\mathcal{K}$ monotone.) By a Hurwitz matrix $M$, we mean, as usual, one for which $\operatorname{Re} \lambda<0$ for any eigenvalue $\lambda$ of $M$, and we denote by $\rho(M)$ the spectral radius of a matrix $M$.

Lemma 1 Let $A=\alpha I-B, \alpha>0$, and assume $B \mathcal{K} \subseteq \mathcal{K}$. Then the following conditions are equivalent:

(1) $A$ is inverse-positive.

(2) $\rho(B)<\alpha$

(3) $-A$ is Hurwitz

Lemma 2 Let $A=\alpha I-B, \alpha>0, B \mathcal{K} \subseteq \mathcal{K}$, and suppose that $A$ is invertible and $A x>0$ for some $x \gg 0$. Then $A$ is inverse-positive.

\section{$4 \quad$ Linear Systems}

Consider a linear system $\dot{x}=A x+B u, y=C x$ that is monotone and admits well defined (and necessarily nondegenerate) I/S and I/O characteristics. We close the system by unity feedback, letting $u=y=C x$, thus forming an autonomous dynamical system. It is easy to compute the $\mathrm{I} / \mathrm{O}$ characteristic from the equation $A x+B u=0$ for a fixed $u \in U$, namely $k(u)=-C A^{-1} B u$. Thus $k^{\prime}(0)=-C A^{-1} B$; this will be important for the statement of the following theorem, which is equivalent to Lemma 6.6 in [2] (see also the remark after the proof).

Theorem 2 Let $\dot{x}=A x+B u, y=C x$, with $A \in \mathbb{R}^{n \times n}, B \in \mathbb{R}^{n \times m}, C \in$ $\mathbb{R}^{m \times n}$, be a linear system that admits an $I / O$ characteristic $k$ and is monotone with respect to cones $\mathcal{K}_{n}$ and $\mathcal{K}_{m}$ in the input-value and state spaces. Assume that $\operatorname{det}\left(I+C A^{-1} B\right) \neq 0$. Then $A+B C$ is Hurwitz iff $-\left(I+C A^{-1} B\right)$ is Hurwitz. In other words, the closed loop system is exponentially stable iff the linear system $\dot{u}=k(u)-u$ is exponentially stable.

Proof. The unity-feedback system is given by $\dot{x}=(A+B C) x$. The hypotheses of monotonicity and existence of characteristic on the linear system are equivalent to the following requirements: i) the positivity cone $\mathcal{K}_{n}$ is positively invariant for the system $\dot{x}=A x$; ii) $B \mathcal{K}_{m} \subseteq \mathcal{K}_{n}$; iii) $C \mathcal{K}_{n} \subseteq \mathcal{K}_{m}$; and iv) $A$ is a Hurwitz matrix. See $[2,8]$ for a proof of this equivalence and other properties 
of monotone (positive) linear systems.

Since $A+B C$ is such that $\dot{x}=(A+B C) x$ is monotone, there exists a PerronFrobenius eigenvalue for $A+B C$, that is, a number $\lambda \in \mathbb{R}$ with maximal real part among the eigenvalues of $A+B C$, and such that $(A+B C) v=\lambda v$ for some $v>0$. (This is standard; see for example Lemma 6.2 of [2].) Also, it holds that $-C A^{-1} B K \subseteq K$, since $k$ is a monotone increasing function. By the Perron-Frobenius theorem there exists a real eigenvalue $\mu=\rho\left(-C A^{-1} B\right)-1$ of $-C A^{-1} B-I$ with maximal real part; the nondegeneracy hypothesis implies that $\mu \neq 0$.

Observe that $A+B C$ is Hurwitz if and only if $\lambda<0$, and $-I-C A^{-1} B$ is Hurwitz if and only if $\mu<0$. So we must prove that $\lambda<0$ if and only if $\mu<0$. By multiplying on both sides by $C A^{-1}$ we obtain : $\left(I+C A^{-1} B\right) C v=\lambda C A^{-1} v$. We prove that $\lambda \neq 0$ : if $\lambda$ were zero, then $\operatorname{det}\left(I+C A^{-1} B\right) \neq 0$ would imply $C v=0$ and $A v=(A+B C) v=0$, contradicting the fact that $A$ is a Hurwitz matrix. Note also that $C v \geq 0$ and $C A^{-1} v=-\int_{0}^{\infty} C e^{A t} v \mathrm{~d} t \leq 0$.

Suppose first that $C v \gg 0$. By continuity of the integrand, we conclude that $C A^{-1} v \ll 0$. If $\lambda<0$, we can apply Lemma 2 (with " $\alpha$ " $=1$, " $B$ " $=-C A^{-1} B$, " $A "=I+C A^{-1} B$, and " $\left.x "=C v\right)$ to conclude that $I+C A^{-1} B$ is inversepositive, and therefore, by Lemma 1 , that $-\left(I+C A^{-1} B\right)$ is Hurwitz.

Conversely, if $-\left(I+C A^{-1} B\right)$ is Hurwitz, then, once again appealing to Lemma 1 , we know that $I+C A^{-1} B$ is inverse-positive. Then, from $\left(I+C A^{-1} B\right)\left(-\lambda^{-1}\right) C v=$ $-C A^{-1} v$, we conclude that $\left(-\lambda^{-1}\right) C v>0$ or $\lambda<0$.

Finally, let us consider the general case, $C v \geq 0$. We show the existence of an $m \times n$ matrix $P$ with $P x \gg 0$ for each $x>0$ : since $\mathcal{K}_{n}$ is closed and pointed, there must be some $(n-1)$-dimensional hyperplane $H \subseteq \mathbb{R}^{n}$ whose intersection with $\mathcal{K}_{n}$ is $\{0\}$. Letting $w \in \mathbb{R}^{n}$ have norm equal to 1 and be perpendicular to $H$, we have without loss of generality $x \cdot w>0$, for every $x>0$. Let now $\mathcal{B}$ be a basis of $\mathbb{R}^{n}$ consisting of an orthonormal basis of $H$, together with $w$. Then $\mathcal{B}$ itself is orthonormal.

We can define a linear transformation $P: \mathbb{R}^{n} \rightarrow \mathbb{R}^{m}$ by freely defining the value of $P$ at each of the elements of $\mathcal{B}$, and we do so by setting $P(w) \gg 0$ and $P(b)=0$ for all other $b \in \mathcal{B}$. Given $x>0$, one can write $x$ as linear combination of the base elements, and the coefficient associated with each $b \in \mathcal{B}$ is $b \cdot x$. Since $P(b)=0$ except for $b=w$, our assertion follows from

$$
P(x)=P((x \cdot w) w)=(x \cdot w) P(w) \gg 0 .
$$

Let now $C_{\epsilon}=C+\epsilon P$, for $\epsilon>0$ small enough so that $\operatorname{det}\left(I+C_{\epsilon} A^{-1} B\right) \neq 0$. Thus we can repeat the procedure above with this new matrix, and we have, 
for $\lambda_{\epsilon}, \mu_{\epsilon}$ denoting the stability modulus of $A+B C_{\epsilon}$ and $-\left(I+C_{\epsilon} A^{-1} B\right)$ respectively, that $\lambda_{\epsilon}<0$ if and only if $\mu_{\epsilon}<0$. By continuity of eigenvalues on both sides of the equivalence under continuous changes in matrix entries, the result follows, taking into account that $\lambda \neq 0, \mu \neq 0$.

The second conclusion of Lemma 6.6 in [2] also holds here in a multidimensional version: since $\lambda \neq 0, \mu \neq 0$, and $\lambda<0$ iff $\mu<0$, it must hold that $\lambda>0$ iff $\mu>0$. That is, there exists a positive eigenvalue of $A+B C$ if and only if there exists a positive eigenvalue of $-\left(I+C A^{-1} B\right)$.

Recall that if a linear system is asymptotically stable, then all of its eigenvalues must have negative real part, and the system is therefore exponentially stable. From the proof of the previous theorem we can deduce a similar property for monotone systems, that we explicitly state below.

Lemma 3 Let $\dot{x}=F(x)$ be a monotone system under some proper cone, $F(\bar{x})=0$, and $\operatorname{det} \frac{\partial F}{\partial x}(\bar{x}) \neq 0$. Then $\bar{x}$ is asymptotically stable if and only if it is exponentially stable.

Proof. We prove the only nontrivial direction: let $\bar{x}$ be an asymptotically stable equilibrium, which implies that the real parts of all eigenvalues of the matrix $A=\operatorname{det} \frac{\partial F}{\partial x}(\bar{x})$ are smaller or equal than zero (see for instance Corollary 5.8.7 of [17]). By monotonicity of $A$, there is a Perron-Frobenius eigenvalue $\lambda$ associated to it, that is, a real eigenvalue such that $\operatorname{Re} \lambda$ is larger than the real part of any other eigenvalue of $A$; see Lemma 6.2 of [2]. But by hypothesis $\lambda \neq 0$ - thus $\lambda<0$ and exponential stability follows.

\section{Proof of Theorem 1}

Now we are ready to prove the main result. In the case that $m=1$, the condition that $\bar{u}$ be a stable equilibrium of the reduced system (3) is equivalent to asking that $k(\bar{u})=\bar{u}$ and $k^{\prime}(\bar{u})-1<0$, since by nondegeneracy $k^{\prime}(\bar{u})-1 \neq$ 0 . This is just the condition $k^{\prime}(\bar{u})<1$ used in [2].

Proof. Much of the proof is identical to that of Theorem 3 in [2]; the supporting Lemmas 6.4 and 6.5 in that paper were actually proven for finite-dimensional inputs. For $\bar{u}$ an equilibrium point of $(3)$, that is $k(\bar{u})=\bar{u}$, let the linearization of the open system around $\bar{u}$ and $k^{X}(\bar{u})$ be denoted as $\dot{x}=A x+B u, y=C x$. The hypotheses of Lemma 3 are satisfied both for $\bar{u}$ in the reduced system and for $\bar{x}=k^{X}(\bar{u})$ in the closed loop system, by nondegeneracy of fixed points in the former, and since $\operatorname{det} A+B C \neq 0$ in the latter; see the proof of Theorem 2 . We therefore have that $\bar{u}$ is asymptotically stable in the reduced system if and only if it is exponentially stable in the reduced system (by Lemma 3), if and 
only if $\bar{x}$ is exponentially stable in the closed loop system (By Theorem 2), if and only if $\bar{x}$ is asymptotically stable in the closed loop system.

The important generic convergence result proven by Hirsch in the late 1980's can be stated in our framework as follows: given an autonomous system that is strongly monotone with respect to some proper cone, almost every initial condition with bounded solution has a limit set contained in the set of equilibria; see Theorem 7.8 in [9]. In the case that the set of equilibria is discrete, as it is here, we can conclude that almost every bounded solution converges to an equilibrium point.

Let $\lambda \in \mathbb{R}$ be the Perron-Frobenius eigenvalue associated with $A+B C$ after linearizing around an unstable equilibrium point $\bar{x}$. By unstability and since we know $\lambda \neq 0$, we have $\lambda>0$; thus $\bar{x}$ is a hyperbolically unstable equilibrium of the closed loop system, and it follows (see [2] and de la Llave et al. [10]) that the set of initial conditions that converge toward $\bar{x}$ in the closed loop system has measure zero. Thus almost all bounded solutions converge to one of the equilibria corresponding to a locally exponentially stable steady state of the reduced system, as stated.

Note that under the present hypotheses merely asymptotic stability is actually ruled out: an equilibrium point is either exponentially stable, or unstable with some eigenvalue with positive real part.

This theorem provides a way to describe the behavior of a complex monotone system in terms of a potentially much simpler, associated system. For $m=2$ a graphical analysis as in $[2,3]$ is also possible, by plotting the vector field $k(u)-u$ on the input space, and observing which equilibria appear to be stable (see example below).

In the case that (3) is itself strongly monotone and has bounded solutions, one can actually apply Hirsch's theorem to it and deduce that almost all trajectories converge toward one of the stable steady states. The question arises as to whether the analogy between the two systems could be carried further: if the output function $h$ were surjective, does it hold that $x\left(t, x_{0}\right)$ converges to $\bar{x}$ if and only if $u\left(t, h\left(x_{0}\right)\right)$ converges to $h(\bar{x})$ ? In other words, do the basins of attraction of each stable point correspond to each other, as the stable points do? Unfortunately this is not true, as the example below will illustrate.

\section{An Example}

We illustrate the main result with an example of a coupled biological circuit. An important class of proteins, referred to as transcription factors, regulate 
transcription of messenger RNA by promoting (or inhibiting) the binding of the enzyme RNA polymerase to the DNA sequence. An autoregulatory transcription factor regulates the production of its own messenger RNA. Transcription factors are very common, and often more than one is necessary for RNA polymerase to initiate transcription. For a mathematical analysis of the simple autoregulatory circuit, see $[15]^{3}$.

Let $p_{1}, p_{2}$ be two autoregulatory transcription factors, and $r_{1}, r_{2}$ their corresponding messenger RNAs. We will couple the circuits by assuming that the proteins are also needed to regulate each other's transcription. The dynamics of the circuit is thus expressed as follows:

$$
\begin{aligned}
& \dot{p}_{i}=a_{i} r_{i}-b_{i} p_{i} \\
& \dot{r}_{i}=g_{i}\left(p_{1}, p_{2}\right)-c_{i} r_{i}
\end{aligned} \quad i=1,2 .
$$

We assume that both $g_{1}\left(p_{1}, p_{2}\right)$ and $g_{2}\left(p_{1}, p_{2}\right)$ are increasing functions of both $p_{1}$ and $p_{2}$, as well as positive and bounded. The interconnections are illustrated in Figure 1. In particular, note that all the solutions of this system are bounded.

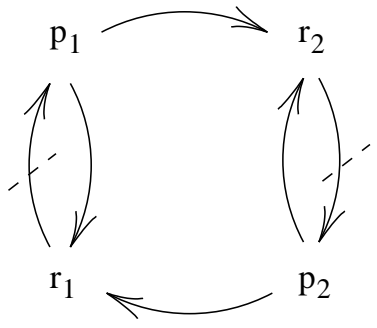

Fig. 1. Interconnections for system 6 . The dotted lines indicate where the interconnections will be cut and replaced by inputs.

We analyse the dynamics of this system by cutting the arcs as indicated in the figure, and we arrive to the following controlled system with two inputs:

$$
\begin{aligned}
& \dot{p}_{i}=a_{i} u_{i}-b_{i} p_{i} \\
& \dot{r}_{i}=g_{i}\left(p_{1}, p_{2}\right)-c_{i} r_{i}
\end{aligned} \quad i=1,2 .
$$

which is monotone under the usual positive orthant cone. If we fix the input

3 The standard model in p. 58 of [15] is in fact another interesting application of Theorem 1: by cutting the arc $x_{n} \rightarrow x_{1}$ as explained in our example, the results in Section 4.2, [15], follow by looking at the fixed points of $k(u)=\alpha_{1}^{-1} \ldots \alpha_{n}^{-1} g(u)$. Furthermore, the local stability of each equilibrium is determined by the slope of $k(u)$ at each corresponding fixed point. 
$\left(u_{1}, u_{2}\right)$, the system converges toward the point $p_{i}=\frac{a_{i}}{b_{i}} u_{i}, r_{i}=\frac{1}{c_{i}} g_{i}\left(\frac{a_{1}}{b_{1}} u_{1}, \frac{a_{2}}{b_{2}} u_{2}\right)$, which constitutes the value of $k^{X}$ at the point $\left(u_{1}, u_{2}\right)$. Note that the arcs cut in Figure 1 are chosen so as to leave the digraph with no directed loops in order for the characteristic to be well defined, while minimizing the number of inputs. Also, two cuts are the minimum since there are two disjoint, directed loops in the digraph. Since we want the closed loop to be (6), we need $h\left(p_{1}, p_{2}, r_{1}, r_{2}\right)=\left(r_{1}, r_{2}\right)$, which when composed with $k^{X}$ yields

$$
k(u, v)=\left(\frac{1}{c_{1}} g_{1}\left(\frac{a_{1}}{b_{1}} u_{1}, \frac{a_{2}}{b_{2}} u_{2}\right), \frac{1}{c_{2}} g_{2}\left(\frac{a_{1}}{b_{1}} u_{1}, \frac{a_{2}}{b_{2}} u_{2}\right)\right) .
$$

Under mass action kinetics assumptions, a quasi-steady state analysis (see [12]) yields for the $g_{i}$ the general form $g_{i}=\hat{\sigma}_{i}\left(p_{1}^{m_{i}} p_{2}^{n_{i}}\right) /\left(\hat{K}_{i}+p_{1}^{m_{i}} p_{2}^{n_{i}}\right)$. The coefficients $m_{i}, n_{i}$ describe the cooperativity with which the proteins bind to the DNA sequence. For instance, if two $p_{1}$ proteins bind to each other (forming a dimer) before acting on the DNA sequence of $p_{i}$, then $m_{i}=2$. It is a reasonable assumption that the cooperativity of a protein is the same for both DNA sequences, that is $m_{1}=m_{2}=m, n_{1}=n_{2}=n$. We set for simplicity $m=2, n=1$. The remaining coefficients $\hat{K}_{i}, \hat{\sigma}_{i}$ are determined by the way the proteins bind to the particular DNA sequence and how they aid the polymerase enzyme. We have $k\left(u_{1}, u_{2}\right)=\left(\sigma_{1}\left(u_{1}^{2} u_{2}\right) /\left(K_{1}+u_{1}^{2} u_{2}\right), \sigma_{2}\left(u_{1}^{2} u_{2}\right) /\left(K_{2}+u_{1}^{2} u_{2}\right)\right)$, where $\sigma_{i}=\hat{\sigma}_{i} c_{i}^{-1}, K_{i}=\hat{K}_{i} a_{1}^{-2} b_{1}^{-2} a_{2}^{-1} b_{2}^{-1}$. Apart from the trivial solution $(0,0)$, the equation $k\left(u_{1}, u_{2}\right)=\left(u_{1}, u_{2}\right)$ can be rewritten as $K_{1}+u_{1}^{2} u_{2}=$ $\sigma_{1} u_{1} u_{2}, \quad K_{2} u_{2}+u_{1}^{2} u_{2}^{2}=\sigma_{2} u_{1}^{2} u_{2}$ We solve for $u_{1}$ in the first equation above and replace in the second equation, obtaining $K_{1} u_{1}^{2}=\left(\sigma_{2} u_{1}^{2}-K_{2}\right)\left(\sigma_{1}-u_{1}\right) u_{1}$. From Figure 2 we see that there might be only one nonnegative solution of

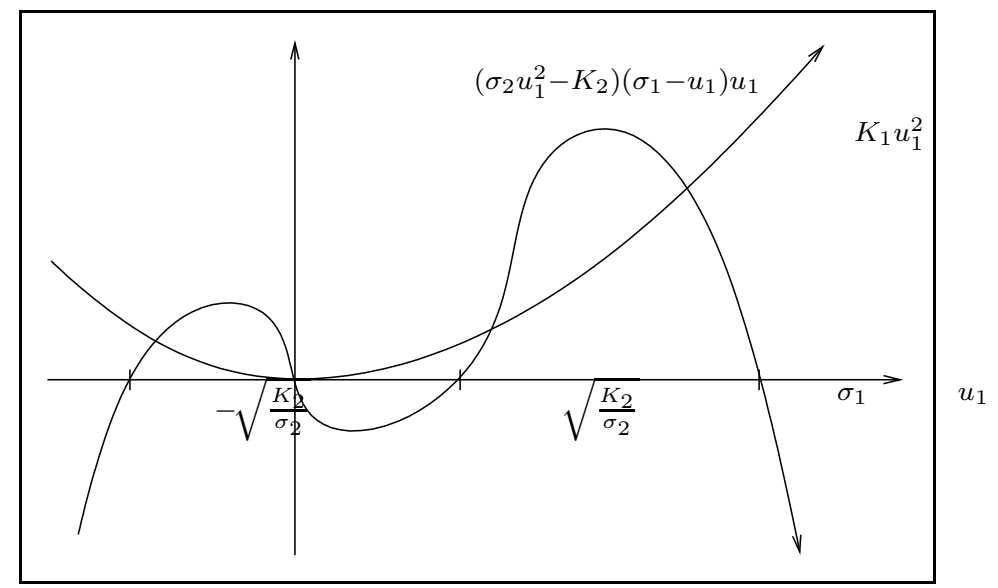

Fig. 2. The solutions of the equation $k\left(u_{1}, u_{2}\right)=\left(u_{1}, u_{2}\right)$

$k\left(u_{1}, u_{2}\right)=\left(u_{1}, u_{2}\right)$ (i.e., the trivial solution $\left.u_{1}=0\right)$, or there may be three nonnegative solutions, in the case that $K_{1}, K_{2}$ are comparatively small. Theorem 1 can be used here to establish a correspondence between these points and the equilibrium points of (6), which sends stable states (of the system 
$\dot{u}=k(u)-I)$ to stable states of $(6)$. Thus, by verifying that there are two stable points and one unstable point in $\dot{u}=k(u)-I$, we will have shown that the same holds for (6). See Figure 3 for an illustration of this in the particular case $\sigma_{1}=4, \sigma_{2}=2, K_{1}=4, K_{2}=5$; note that additional solutions may appear outside of the positive quadrant.

Given the simple form of the output function $h(x)=\left(r_{1}, r_{2}\right)$, any basin of attraction of $\dot{u}=k(u)-I$ will correspond in $X$ (under $h^{-1}$ ) to a rather rigid set, namely that of every $\left(p_{1}, p_{2}, r_{1}, r_{2}\right)$ such that $\left(r_{1}, r_{2}\right)$ is in the basin. It is clear that the basins of attraction of (6) don't have this form - this limits the analogy between (6) and its reduced system.

On the other hand, the same procedure can be applied for cones that are not necessarily the positive orthant: for instance if, in the above example, each protein promoted its own growth and inhibited each other's growth, then $\mathcal{K}_{n}=\mathbb{R}^{+} \times \mathbb{R}^{-} \times \mathbb{R}^{+} \times \mathbb{R}^{-}$would make (6) strongly monotone.

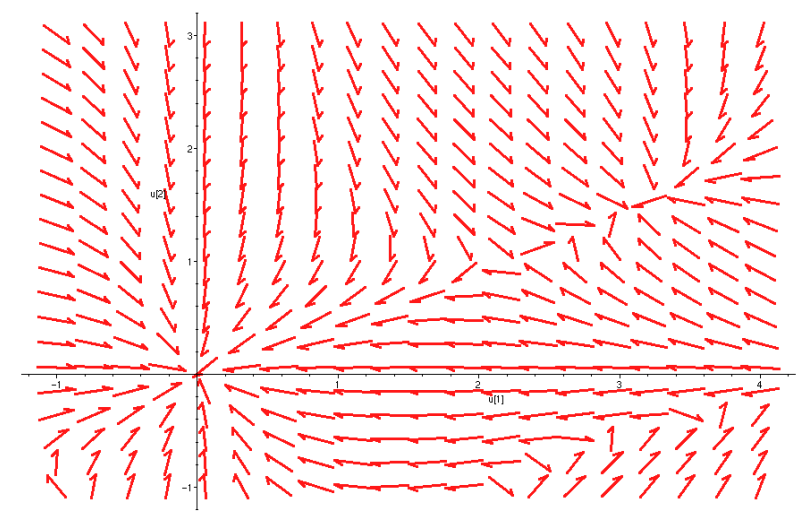

Fig. 3. The vector field $\gamma(u)=k(u)-I$, using parameter values $\sigma_{1}=4, \sigma_{2}=2$, $K_{1}=4, K_{2}=5$.

\section{Cascades and Strong Monotonicity}

In the paper [2], it is shown that a monotone controlled system which is weakly transparent and strongly excitable, or strongly transparent and weakly excitable, has a closed loop which is strongly monotone (definitions below). This is used as a convenient sufficient condition for the corresponding hypothesis of the main result, here Theorem 1. One drawback of the setup of that paper is that it is not robust under cascading, in the sense that a cascade of weakly transparent and excitable systems is not necessarily weakly transparent nor weakly excitable. In what follows, we strenghten these definitions so as to address this point. One possible application is the introduction of "pseudo-delays" in the system by adding a cascade of systems of the form $\tau \dot{z}_{i}=v_{i}-z_{i}, v_{i+1}=z_{i}, i=1,2, \ldots$ Note that this cascade respects both the 
monotonicity and the characteristic of the original system. The introduction of true delays in the loop also does not affect our conclusions; this can be shown by appealing to Corollary 5.5.2 in Smith [15], which asserts that the stability properties of equilibria in a strongly monotone delay system do not change when the delays are ignored. See [7] for further discussion.

We say that a system of the form (1) is sign definite if for each $i \neq j$, it holds that $\frac{\partial f_{i}}{\partial x_{j}}(x, u) \bowtie 0$ for all $(x, u) \in X \times U$, where the relation $\bowtie$ stands for either " $<"$, " ">", or "=", and of course different signs are allowed for different pairs $(i, j)$. Similarly for partial derivatives $\frac{\partial f_{i}}{\partial u_{j}}(x, u)$ for all $i, j$, and for partial derivatives of $h$. One can then define a signed digraph by using the input and the state variables as nodes, as usual. It can be shown that a sign definite autonomous system is monotone with respect to some orthant cone iff every undirected cycle in its digraph has an even number of "-"'s, and that in that case, it is strongly monotone if the digraph is strongly connected. See [2].

In what follows, we say that a monotone system (1) is partially excitable if for any $x_{1} \leq x_{2}$, arbitrary inputs $u_{1}, u_{2}$, and any $t_{0}>0$, the following properties hold: 1) $u_{1}<u_{2}$ a.e. on $\left(0, t_{0}\right)$ implies $x\left(t, x_{1}, u_{1}\right)<x\left(t, x_{2}, u_{2}\right), t \in\left(0, t_{0}\right)$, and 2) $u_{1} \ll u_{2}$ a.e. on $\left(0, t_{0}\right)$ implies $x\left(t, x_{1}, u_{1}\right) \ll x\left(t, x_{2}, u_{2}\right), t \in\left(0, t_{0}\right)$. We also say that (1) is strongly excitable if $u_{1}<u_{2}$ a.e. on $\left(0, t_{0}\right)$ implies $x\left(t, x_{1}, u_{1}\right) \ll x\left(t, x_{2}, u_{2}\right), t \in\left(0, t_{0}\right)$. Further, we will say that (1) is partially transparent if for arbitrary inputs $u_{1} \leq u_{2}$ and initial conditions $x_{1}, x_{2}$ one has 1) $x_{1}<x_{2}$ implies $h\left(x\left(t, x_{1}, u_{1}\right)\right)<h\left(x\left(t, x_{2}, u_{2}\right)\right)$, and 2) $x_{1} \ll x_{2}$ implies $h\left(x\left(t, x_{1}, u_{1}\right)\right) \ll h\left(x\left(t, x_{2}, u_{2}\right)\right)$. It is strongly transparent if $x_{1}<x_{2}$ implies $h\left(x\left(t, x_{1}, u_{1}\right)\right) \ll h\left(x\left(t, x_{2}, u_{2}\right)\right)$, for all $t>0$ for which the solutions $x\left(t, x_{i}, u_{i}\right)$ are defined. Note that the first condition for partial excitability and the second condition for partial transparency correspond to the notions of weak excitability and weak transparency, respectively, in the terminology of [2] (borrowed from [11]).

In particular partial excitability (transparency) implies weak excitability (transparency). But the converse is not true: in the cooperative case, if there are arcs from a fixed input to every single state, but no arcs from other inputs whatsoever, then the system is weakly excitable but not partially excitable since $u_{1}<u_{2}$ doesn't imply $x\left(t, \xi, u_{1}\right)<x\left(t, \xi, u_{2}\right)$. Similarly for transparency. The valid implication allows us nevertheless to quote Theorem 2 from [2] in our present terminology:

Proposition 1 A monotone system (1) that is partially excitable and partially transparent has strongly monotone feedback loop provided that it is also either strongly excitable or strongly transparent.

It has also been shown in [2] that in the case of orthant cones and sign definite systems, there are simple conditions on the digraph of the system that 
imply transparency and excitability statements. For instance, if there exists a directed path from every input variable (from every state variable) to every state variable (to every output variable), then the system is strongly excitable (strongly transparent). We show a similar result for the definitions above, which complements Theorems 4 and 5 of [2].

Lemma 4 Let (1) be a sign definite controlled system that is monotone under some orthant cone. If from every input (from every state) there exists a path towards some state (towards some output), and if towards every state (towards every output) there exists a path from some input (from some state), then the system is partially excitable (partially transparent).

Proof. These results follow from an adaptation of the proofs of Theorems 4 and 5 in Appendix A of [2]. Consider first partial excitability: by Case 2 of Lemma A1 of [2], since every input variable $u_{j}$ reaches some $x_{i}$ through a directed path, $u_{1}<u_{2}$ a.e. on $\left(0, t_{0}\right)$ implies that $x\left(t, \xi, u_{1}\right)<x\left(t, \xi, u_{2}\right)$ for any $\xi, t \in\left(0, t_{0}\right)$. By monotonicity, $x\left(t, x_{1}, u_{1}\right) \leq x\left(t, x_{2}, u_{1}\right)<x\left(t, x_{2}, u_{2}\right)$. As to the second assertion, the proof given for Theorem 4 in [2] actually shows that if every $x_{i}$ is reachable from some $u_{j}$, then for any $\xi: u_{1} \ll u_{2} \Rightarrow$ $x\left(t, \xi, u_{1}\right) \ll x\left(t, \xi, u_{2}\right)$. The statement for $x_{1} \leq x_{2}$ follows by monotonicity. A similar argument is valid for transparency: given an input $u$ and assuming $\xi_{1}<\xi_{2}$, there is $i$ such that $\left\{t \geq 0 \mid x_{i}\left(t, \xi_{1}, u\right)<x_{i}\left(t, \xi_{2}, u\right)\right\} \cap[0, \epsilon)$ has nonzero measure for every $\epsilon>0$, see sketch of proof of Theorem 5 in [2]. If $y_{i}$ is reachable from $x_{i}$, then $h_{j}\left(x\left(t, \xi_{1}, u\right)\right)<h_{j}\left(x\left(t, \xi_{2}, u\right)\right), t>0$. The statement for $u_{1} \leq u_{2}$ follows by monotonicity, and from the fact that every $x_{i}$ reaches some $y_{j}$. By the same token, since every $y_{j}$ is reached by some $x_{i}, x_{1} \ll x_{2}$ implies $h_{j}\left(x\left(t, x_{1}, u_{1}\right)\right) \ll h_{j}\left(x\left(t, x_{2}, u_{2}\right)\right), t>0$.

Now consider, instead of system (1), a cascade of the form

$$
\dot{x}^{i}=f_{i}\left(x^{i}, u^{i}\right), u^{i+1}=h_{i}\left(x^{i}\right), i=1 \ldots N
$$

We will refer to the subsystem $\dot{x}^{i}=f_{i}\left(x^{i}, u^{i}\right), u^{i+1}=h_{i}\left(x^{i}\right)$, as $(8 . i)$.

Lemma 5 Suppose that the cascade system (8) is monotone, and that each subsystem (8.i) is both partially excitable and partially transparent. Then (8) is partially excitable and partially transparent. Further, if (8.1) is strongly excitable, then (8) is strongly excitable. If (8.N) is strongly transparent, then (8) is strongly transparent.

Proof. Consider any pair of initial conditions $x_{1}=\left(x_{1}^{i}\right)_{i}<x_{2}=\left(x_{2}^{i}\right)_{i}$ of the closed loop system, and let $x_{1}^{i}(t), x_{2}^{i}(t), i=1 \ldots N$ be the corresponding induced inputs, $u_{j}^{2}(t)=h_{1}\left(x_{j}^{1}(t)\right), \ldots, u_{j}^{N}(t)=h^{N-1}\left(x_{j}^{N-1}(t)\right), u_{j}^{1}(t)=u_{j}^{N+1}(t)=$ $h_{N}\left(x_{j}^{N}(t)\right), j=1,2$, the corresponding outputs on a maximally defined interval (from now on we will restrict ourselves to this interval). In particular, 
note that $x_{j}^{i}(t)$ is the solution of the open system $\dot{x}^{i}=f\left(x^{i}, u_{j}^{i}\right)$ with initial condition $x_{j}^{i}$ and input $u_{j}^{i}(\cdot), j=1,2, i=1 \ldots N$. The monotonicity of $(8)$ is clear since it is the closed loop of a cascade of monotone systems, under positive feedback. By monotonicity we thus have $x_{1}^{i}(t) \leq x_{2}^{i}(t)$, and consequently $u_{1}^{i}(t) \leq u_{2}^{i}(t)$, for every $t \geq 0$.

We prove the partial excitability of the cascade: if $u_{1}^{1}<u_{2}^{1}$ on some interval $\left(0, t_{0}\right)$, then $x_{2}^{1}<x_{2}^{1}$ on that interval by partial excitability of (8.1). But this in particular implies that $x_{1}(t) \neq x_{2}(t)$ on this interval, hence $x_{1}(t)<x_{2}(t)$. If, on the other hand, $u_{1}^{1} \ll u_{2}^{1}$ on $\left(0, t_{0}\right)$, then $x_{2}^{1} \ll x_{2}^{1}$ on that interval by partial excitability of (8.1), $u_{1}^{2} \ll u_{2}^{2}$ by partial transparency of (8.1), and so on, so that by induction $x_{1}^{i}(t) \ll x_{2}^{i}(t)$ for all $i$ by partial excitability of $(8 . i)$ for every $i$, and so $x_{1} \ll x_{2}$. The proof for partial transparency is very similar.

Now suppose that $(8 . i)$ is strongly excitable, and let $u_{1}^{1}<u_{2}^{1}$. By strong excitability we have $x_{1}^{1} \ll x_{2}^{1}$, and by partial transparency and excitability $x_{1}^{2}(t) \ll x_{2}^{2}(t)$. Continuing as before, we have $x_{1}(t) \ll x_{2}(t)$. The last assertion is proven in a similar way.

Corollary 1 Let the system (8) be monotone and let each (8.i) be partially excitable and partially transparent. Let one of these two conditions be also strong, for some (8.i). Then the closed loop system obtained by setting $u^{1}=$ $u^{N+1}$ in (8) is strongly monotone.

Proof. By the previous lemma, the cascade (8) is itself partially excitable and partially transparent. If (8.1) is strongly excitable or if $(8 . N)$ is strongly transparent, the conclusion follows by the previous lemma and the previous proposition. If $(8 . i)$ is strongly excitable for $i>1$, change the order of the cascade by eliminating $(8.1) \ldots(8 . i-1)$ and appending them after $(8 . N)$ in the natural way, Note that the closed loop system remains the same, and apply the previous argument. A similar proof applies to the second statement of the Corollary.

\section{Acknowledgements}

We thank David Angeli for many helpful comments, Adam Halasz for the suggestion to point out that "pseudo-delays" in the feedback loop do not change our conclusions, and Hal Smith for pointing us to Corollary 5.5.2 in [15].

\section{References}

[1] D. Angeli, E.D. Sontag, Monotone control systems, IEEE Trans. Autom Control 48 (2003) 1684-1698.

[2] D. Angeli, E.D. Sontag, Multistability in monotone input/output systems, Systems and Control Letters 51 (2004) 185-202. 
[3] D. Angeli, J.E. Ferrell, Jr., E.D. Sontag, Detection of multi-stability, bifurcations, and hysteresis in a large class of biological positive-feedback systems, Proceedings of the National Academy of Sciences USA 101 (2004) $1822-1827$.

[4] D. Angeli, E.D. Sontag, Interconnections of monotone systems with steady-state characteristics, in Optimal Control, Stabilization, and Nonsmooth Analysis (de Queiroz, M., M. Malisoff, and P. Wolenski, eds.), Springer-Verlag, Heidelberg, 2004, to appear.

[5] A.C. Antoulas, Lectures on the Approximation of Large-Scale Dynamical Systems, SIAM, Philadelphia, 2004, to appear.

[6] A. Berman, R.J. Plemmons, Nonnegative Matrices in the Mathematical Sciences, Academic Press, New York, 1979.

[7] G.A. Enciso, E.D. Sontag, A Remark on Multistability for Monotone Systems, 43rd IEEE Conference on Decision and Control, 2004, to appear.

[8] L. Farina and S. Rinaldi, Positive Linear Systems, Wiley-Interscience, Hoboken, NJ, 2000.

[9] M.W. Hirsch, Stability and convergence in strongly monotone dynamical systems, Reine und Angew. Math. 383 (1988) 1-53.

[10] R. de la Llave, C.E. Wayne, On Irwin's proof of the pseudostable manifold theorem, Math Z. 219 (1995) 301-321.

[11] C. Piccardi, S. Rinaldi, Excitability, stability and the sign of equilibria in cooperative systems, Syst. Control Lett. 46 (2002) 153-163.

[12] J.Keener, J. Sneyd, Mathematical Physiology, New York, Springer, 1998.

[13] H. Kunze, D. Siegel, Monotonicity with respect to Closed Convex Cones I, Dyn. of Cont., Disc. and Imp. Sys. 5 (1999) 433-449.

[14] H. Schneider, M. Vidyasagar, Cross positive matrices. SIAM J. Numer. Anal. 7 (1970) 508-519,

[15] Hal L. Smith, Monotone Dynamical Systems, AMS, Providence, R.I. 1995.

[16] M.W.Hirsch, H.L. Smith, Competitive and cooperative systems: a mini-review. Positive Systems, POSTA 2003, Springer, 2003.

[17] E.D. Sontag, Mathematical Control Theory, Springer Verlag, New York, 1998.

[18] E.D. Sontag, Some new directions in control theory inspired by systems biology, Systems Biology (2004) 9-18.

[19] M. Vajta, Some remarks on Padé approximations, $3^{\text {rd }}$ TEMPUS-INTCOM Symposium, Veszprém, Hungary (2000).

[20] P. Volkmann, Gewoehnliche Differentialgleichungen mit quasimonoton wachsenden Funktionen in topologischen Vektorraeumen, Math. Z. 127 (1972) 157-164. 